\title{
Phenol-Directed Enantioselective Allylation of Aldimines and Ketimines
}

\author{
Philippe M. A. Rabbat, S. Corey Valdez, and James L. Leighton \\ Department of Chemistry, Columbia University, New York, New York 10027 \\ Supporting Information
}

General Information. All reactions were carried out under an atmosphere of nitrogen in flame- or oven-dried glassware with magnetic stirring unless otherwise indicated. Degassed solvents were purified by passage through an activated alumina column. Absolute ethanol was purchased from Pharmco Inc. and used without purification. 2-Aminophenol, $(1 S, 2 S)$-pseudoephedrine and all aldehyde reagents were purchased from Aldrich. Aromatic aldehydes were purified by passage through basic alumina immediately before use. Aliphatic aldehydes were distilled before use and stored at $-20{ }^{\circ} \mathrm{C}$. ${ }^{1} \mathrm{H}$ NMR spectra were recorded on a Bruker spectrometer of the field strength indicated in the experimentals at 25 ${ }^{\circ} \mathrm{C}$ and are reported in ppm from TMS internal standard $(0.0 \mathrm{ppm})$. Data are reported as follows: ( $\mathrm{s}=$ singlet, br $\mathrm{s}=$ broad singlet, $\mathrm{d}=$ doublet, $\mathrm{t}=$ triplet, $\mathrm{q}=$ quartet, quin=quintet, $\mathrm{m}=$ multiplet, $\mathrm{dd}=\mathrm{doublet}$ of doublets, td= triplet of doublets; coupling constant(s) in Hz; integration; assignment). Proton decoupled ${ }^{13} \mathrm{C}$ NMR spectra were recorded on a Bruker spectrometer at the indicated field strength at $25{ }^{\circ} \mathrm{C}$ and are reported in ppm from $\mathrm{CDCl}_{3}$ internal standard $(77.0 \mathrm{ppm})$. Infrared spectra were recorded on a Perkin Elmer Paragon 1000 FT-IR spectrometer. Mass spectra were recorded on a JEOL LCmate spectrometer. Optical rotations were recorded on a Jasco DIP-1000 digital polarimeter.

General procedure for the allylation and crotylation of imines of aliphatic aldehydes $(\operatorname{Method} A$, Scheme 2): To a stirred suspension of 2-amino- $m$-cresol $(1 \mathrm{mmol})$ and $3 \AA$ molecular sieves $(\sim 0.5 \mathrm{~g})$ in $\mathrm{CH}_{2} \mathrm{Cl}_{2}$ is added the aldehyde $(1.02 \mathrm{mmol})$. The resulting suspension is stirred for $1-3 \mathrm{~h}$, and then filtered with $\mathrm{CHCl}_{3}$ washes. The filtrate is concentrated and the residue is treated with $\mathrm{PhCH}_{3}$ and the resulting solution is concentrated. The resulting unpurified imines are dissolved in $\mathrm{Et}_{2} \mathrm{O}(10 \mathrm{~mL})$, and the requisite silane reagent $((S, S)-\mathbf{1},(S, S)-\mathbf{4}$, or $(S, S)-\mathbf{5})$ is added. After $20 \mathrm{~h}$ the resulting solution is quenched by the addition of $\mathrm{MeOH}(1 \mathrm{~mL})$, and the resulting mixture is concentrated. The residue is partitioned between EtOAc and water, the layers are separated and the aqueous layer is extracted twice with EtOAc. The combined organic layers are washed with water, then brine, dried over $\mathrm{MgSO}_{4}$, filtered and concentrated. The residue is purified by flash column chromatography to afford the pure products.

(R)-2-(1-cyclohexylbut-3-enylamino)-3-methylphenol (3): After reaction and workup as in the general procedure above, the title compound was isolated as a clear oil (211.9mg, 82\%). ${ }^{1} \mathrm{H}$ NMR (300 
$\left.\mathrm{MHz}_{\mathrm{CDCl}}\right) \delta 6.89$ (d, $\left.J=7.7 \mathrm{~Hz}, 1 \mathrm{H}, \mathrm{Ar}-\mathbf{H}\right), 6.74-6.69(\mathrm{~m}, 1 \mathrm{H}), 6.85-6.50$ (br s, $1 \mathrm{H}, \mathrm{N}-\mathbf{H}$ or O-H) 6.66-6.61 (m, 1H, Ar-H), 5.88-5.73 (m, 1H, $\left.\mathrm{CH}_{2}-\mathrm{CH}=\mathrm{CH}_{2}\right)$, 5.14-5.05 (m, 2H, $\mathrm{CH}_{2}-\mathrm{CH}=\mathrm{CH}$ ), 2.90-2.83 (m, 1H, $c$-Hex-CH-NH), 2.99-2.45 (br s, $1 \mathrm{H}, \mathrm{N}-\mathbf{H}$ or $\mathrm{O}-\mathbf{H}), 2.30-2.18\left(\mathrm{~m}, 1 \mathrm{H}\right.$, one of $\left.\mathrm{CH}_{2}-\mathrm{CH}_{2} \mathrm{CH}_{2}\right), 2.24$ (s, 3H, Me-Ar), 2.12-2.00 (m, 1H, one of $\left.\mathrm{CH}_{2}-\mathrm{CH}=\mathrm{CH}_{2}\right), 1.88-1.56(\mathrm{~m}, 5 \mathrm{H}, c-\mathrm{Hex}$ protons), 1.52-1.40 (m, 1H, $c$-Hex proton), 1.34-1.06 (m, 5H, $c$-Hex protons); ${ }^{13} \mathrm{C} \mathrm{NMR}\left(75 \mathrm{MHz}, \mathrm{CDCl}_{3}\right) \delta 152.8,136.3$, $133.5,132.1,125.0,122.0,117.1,112.1,62.1,40.5,35.7,28.8,26.8,26.7,18.2$. IR (thin film) 3600-2500 (br s), 3075 (m), 2925 (s), 2853 (s), 1639 (w), 1615 (w), 1588 (m), 1474 (s), 1460 (s), 1345 (m), 1276 (s), 1232 (s), 1180 (m), 995 (m), $914(\mathrm{~m}), 773 \mathrm{~cm}^{-1}$ (s); MS (CI+) calculated for $\mathrm{C}_{17} \mathrm{H}_{25} \mathrm{NO}$ : 259, observed: $260(\mathrm{M}+1) ;[\alpha]_{\mathrm{D}}=-5.6^{\circ}\left(c 1.71, \mathrm{CHCl}_{3}\right)$.

(S)-3-methyl-2-(1-phenylhex-5-en-3-ylamino)phenol (6): After reaction and workup as in the general procedure above, the title compound was isolated by flash chromatography (3-8\% EtOAc/hex) as a clear oil $(111.6 \mathrm{mg}, 40 \%) .{ }^{1} \mathrm{H}$ NMR $\left(300 \mathrm{MHz}, \mathrm{CDCl}_{3}\right) \delta 7.28-7.08(\mathrm{~m}, 5 \mathrm{H}, \mathrm{Ph}-\mathrm{H}), 6.92(\mathrm{t}, J=7.9 \mathrm{~Hz}$, 1H, Ar-H), 6.74 (br d, $J=7.9 \mathrm{~Hz}, 1 \mathrm{H}, \mathrm{Ar}-\mathbf{H}), 6.65$ (m, 1H, Ar-H), 5.92-5.77 (m, 1H, $\left.\mathrm{CH}_{2}-\mathrm{CH}_{\mathbf{H}} \mathrm{CH}_{2}\right)$, 5.20-5.12 (m, 2H, $\mathrm{CH}_{2}-\mathrm{CH}=\mathrm{CH}_{2}$ ), 3.08 (quint, $\left.J=6.1 \mathrm{~Hz}, 1 \mathrm{H}, \mathrm{CH}_{2}-\mathrm{CH}(\mathrm{NH}) \mathrm{CH}_{2}\right), 2.75-2.57(\mathrm{~m}, 2 \mathrm{H}$, $\left.\mathrm{PhCH}_{2} \mathrm{CH}_{2}\right), 2.41-2.10\left(\mathrm{~m}, 2 \mathrm{H}, \quad \mathrm{CH}_{2}-\mathrm{CH}=\mathrm{CH}_{2}\right), 2.19\left(\mathrm{~s}, 3 \mathrm{H}, \quad \mathrm{NHAr}-\mathrm{CH}_{3}\right) 1.82-1.72 \quad(\mathrm{~m}, 2 \mathrm{H},-$ $\left.\mathrm{CH}_{2} \mathrm{CH}_{2} \mathrm{CH}(\mathrm{NH}) \mathrm{CH}_{2}-\right) ;{ }^{13} \mathrm{C}$ NMR $\left(75 \mathrm{MHz}, \mathrm{CDCl}_{3}\right) \delta 153.1,145.8,135.1,134.2,131.3,128.5,128.4$, 126.0, 125.6, 122.0, 118.5, 112.0, 56.4, 39.2, 35.9, 32.2, 18.1; IR (thin film) 3600-2500 (br, s), 3062 (s), 3026 (s), 2939 (s), 2859 (m), 1639 (w), 1588 (s), 1484 (s), 1554 (s), 1345 (m), 1278 (s), 1233 (s), 1089 (m), 996 (w), 917 (m), 773 (s), 745 (s), $699 \mathrm{~cm}^{-1}$ (s); MS (CI+) calculated for $\mathrm{C}_{19} \mathrm{H}_{23} \mathrm{NO}$ : 281, observed: $282(\mathrm{M}+1) ;[\alpha]_{\mathrm{D}}=+33.8^{\circ}\left(c 0.991, \mathrm{CHCl}_{3}\right)$.

2-((1S,2R)-1-cyclohexyl-2-methylbut-3-enylamino)-3-methylphenol (13): After reaction and workup as in the general procedure above, the title compound was purified by flash chromatography (3$6 \% \mathrm{EtOAc} / \mathrm{hex})$ and isolated as a clear oil $(174 \mathrm{mg}, 64 \%) .{ }^{1} \mathrm{H} \mathrm{NMR}\left(300 \mathrm{MHz}, \mathrm{CDCl}_{3}\right) \delta 6.79(\mathrm{t}, J=$ 7.7Hz, 1H, Ar-H), 6.69-6.59 (m, 2H, Ar-H), 6.24-5.97 (br s, 1H, N-H or O-H), 5.97-5.84 (m, 1H, $\left.\mathrm{CH}\left(\mathrm{CH}_{3}\right)-\mathrm{CH}=\mathrm{CH}_{2}\right), 5.10-5.02\left(\mathrm{~m}, 2 \mathrm{H}, \mathrm{CH}\left(\mathrm{CH}_{3}\right)-\mathrm{CH}=\mathrm{CH}_{2}\right), 3.01(\mathrm{t}, J=4.8 \mathrm{~Hz}, 1 \mathrm{H}, c$-Hex-CH-(NHAr)$\left.\mathrm{CH}\left(\mathrm{CH}_{3}\right)-\mathrm{CH}=\mathrm{CH}_{2}\right), 3.12-2.66$ (br s, $1 \mathrm{H}, \mathrm{N}-\mathrm{H}$ or $\left.\mathrm{O}-\mathrm{H}\right), 2.43$ (sextet, $J=6.5 \mathrm{~Hz}, 1 \mathrm{H}, \mathrm{CH}\left(\mathrm{CH}_{3}\right)-\mathrm{CH}_{=} \mathrm{CH}_{2}$ ), $2.16\left(\mathrm{~s}, 3 \mathrm{H}, \mathrm{Ar}-\mathrm{CH}_{3}\right), 1.84-1.35(\mathrm{~m}, 6 \mathrm{H}, c$-Hex protons), 1.28-1.05 (m, 5H, $c$-Hex protons), 1.10 (d, $J=$ $\left.7.1 \mathrm{~Hz}, \mathrm{CH}\left(\mathrm{CH}_{3}\right)-\mathrm{CH}=\mathrm{CH}_{2}\right) ;{ }^{13} \mathrm{C} \mathrm{NMR}\left(75 \mathrm{MHz}, \mathrm{CDCl}_{3}\right) \delta 150.4,142.0,133.8,131.2,123.0,122.7$, 115.2, 112.7, 65.0, 41.3, 40.1, 30.4, 29.9, 26.8, 26.7, 18.8, 17.4; IR (thin film) 3600-2800 (br s), 2926 (s), 2853 (s), 1587 (m), 1488 (s), 1450 (s), 1277 (m), 1233 (m), 1091 (w), 1004 (w), 949 (w), 913 (w) 768 
(m), $741 \mathrm{~cm}^{-1}$; MS $(\mathrm{CI}+)$ calculated for $\mathrm{C}_{18} \mathrm{H}_{27} \mathrm{NO}: 273$, observed: $274(\mathrm{M}+1) ;[\alpha]_{\mathrm{D}}=+29.8^{\circ}(c$ 1.46, $\left.\mathrm{CHCl}_{3}\right)$.

General procedure for the preparation of imines of aromatic aldehydes (Scheme2): To a stirred suspension of 2-aminophenol ( $1 \mathrm{mmol})$ in $\mathrm{EtOH}(5 \mathrm{ml} / \mathrm{mmol}$ of 2-aminophenol) is added freshly purified aldehyde $(1.02 \mathrm{mmol})$. The resulting solution is stirred for $1-5 \mathrm{~h}$ at room temperature and concentrated. The residue is treated with $\mathrm{PhCH}_{3}$ and the resulting solution is concentrated to give the imines, which were typically pure enough for use without further purification.

General procedure for the allylation and crotylation of aromatic aldehyde-derived imines (Method B, Scheme 2): To a solution of the imine (1.0 mmol) in $\mathrm{CH}_{2} \mathrm{Cl}_{2}(10 \mathrm{~mL})$ at ambient temperature is added the requisite silane reagent $((S, S)-\mathbf{1},(S, S)-\mathbf{4}$, or $(S, S)-\mathbf{5})(1.2 \mathrm{mmol})$. The resulting solution is stirred for $16 \mathrm{~h}$ then quenched by the addition of $\mathrm{MeOH}(1 \mathrm{~mL})$. The mixture is concentrated, and the residue partitioned between EtOAc and water. The layers are separated, and the aqueous layer extracted twice with EtOAc. The combined organic layers are washed with water, then brine, dried over $\mathrm{MgSO}_{4}$, filtered, and concentrated. The residue is purified by flash column chromatography to afford pure products.

(R)-2-(1-phenylbut-3-enylamino)phenol (8): Purified by flash chromatography (10-20\% EtOAc/hexanes) to give $198 \mathrm{mg}(83 \%)$ of the title compound as a dark solid. ${ }^{1} \mathrm{H}$ NMR (300 $\left.\mathrm{MHz}, \mathrm{CDCl}_{3}\right)$ ठ 7.36-7.25 (m, 3H, Ph-H), 7.23-7.16 (m, 2H, Ph-H), 6.70-6.61 (br m, 2H, Ar-H), 6.53 (br d, J = 7.5Hz, $1 \mathrm{H}, \mathrm{Ar}-\mathbf{H}), 6.36$ (br d, $J=8.0 \mathrm{~Hz}, 1 \mathrm{H}, \mathrm{Ar}-\mathbf{H}), 5.85-5.69\left(\mathrm{~m}, 1 \mathrm{H}, \mathrm{CH}_{2}-\mathrm{CH}=\mathrm{CH}_{2}\right)$, 5.22-5.08 (m, 2H, $\mathrm{CH}_{2}-$ $\mathrm{CH}=\mathrm{CH}_{2}$ ), 5.20-4.80 (br s, $1 \mathrm{H}, \mathrm{N}-\mathbf{H}$ or O-H), 4.64-4.24 (br s, $1 \mathrm{H}, \mathrm{N}-\mathbf{H}$ or O-H), 4.35 (br t, $J=6.6 \mathrm{~Hz}$, $1 \mathrm{H})$, 2.68-2.48 (m, 2H, $\left.\mathrm{CH}_{2}-\mathrm{CH}=\mathrm{CH}_{2}\right) ;{ }^{13} \mathrm{C} \mathrm{NMR}\left(75 \mathrm{MHz}, \mathrm{CDCl}_{3}\right) \delta 143.5,136.1,134.6,128.6,127.0$, 126.5, 121.6, 118.3, 117.8, 114.5, 113.8, 57.9, 43.1; IR (thin film) 3700-2700 (br, s), 3062 (s), 1640 (m), 1611 (s), 1514 (s), 1452 (s), 1271 (s), 922 (s), 741 (s), 702 (s) cm cm $^{-1}$ MS (CI+) calculated for $\mathrm{C}_{16} \mathrm{H}_{17} \mathrm{NO}$ : 239, found: $239(\mathrm{M}+\mathrm{H}) ;[\alpha]_{\mathrm{D}}=-12^{\circ}\left(c 1.59, \mathrm{CHCl}_{3}\right)$.

(R)-2-(1-o-tolylbut-3-enylamino)phenol (9): Isolated by flash chromatography (7.5-17.5\% EtOAc/hexanes) which yielded the title compound as an orange solid (226 mg, 89\%). ${ }^{1} \mathrm{H}$ NMR (300

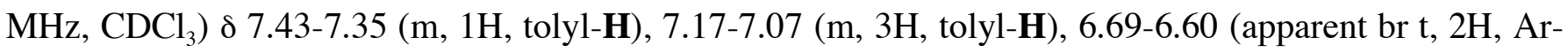
H), 6.56-6.48 (apparent t, 1H, Ar-H), 6.26-6.21 (apparent d, 1H, Ar-H), 5.88-5.73 (m, 1H, $\mathrm{CH}_{2}-$ $\left.\mathrm{CH}=\mathrm{CH}_{2}\right), 5.24-5.10\left(\mathrm{~m}, 2 \mathrm{H}, \mathrm{CH}_{2}-\mathrm{CH}=\mathrm{CH}_{2}\right), 4.85-4.60$ (br s, $1 \mathrm{H}, \mathrm{N}-\mathbf{H}$ or O-H), 4.60-4.52 (m, $1 \mathrm{H}$ tolCH-NH), 4.52-4.30 (br s, $1 \mathrm{H}, \mathrm{N}-\mathrm{H}$ or $\mathrm{O}-\mathbf{H}), 2.73$ (m, $2 \mathrm{H}, \mathrm{CH}_{2}-\mathrm{CH}=\mathrm{CH}_{2}$ ), 2.43 (s, 3H CH $\mathrm{C}_{6} \mathrm{H}_{4} \mathrm{CHNH}$ ); 
${ }^{13} \mathrm{C} \mathrm{NMR}\left(75 \mathrm{MHz}, \mathrm{CDCl}_{3}\right) \delta 143.51,141.2,136.1,134.8,134.7,130.6,126.8,126.5,125.6,121.6,118.3$, 117.6, 114.4, 113.2, 53.9, 41.4, 19.2; IR (thin film) 3600-2500 (br s), 3424 (s), 3073 (m), 2977 (m), 2926 (m), 1639 (w), 1611 (s), 1511 (s), 1445 (s), 1271 (s), 1100 (m), 911 (m), $737 \mathrm{~cm}^{-1}$; MS (CI+) calculated for $\mathrm{C}_{17} \mathrm{H}_{19} \mathrm{NO}$ : 253, observed: $254(\mathrm{M}+1) ;[\alpha]_{\mathrm{D}}=-23.7^{\circ}\left(c 1.79, \mathrm{CHCl}_{3}\right)$.

(R)-2-(1-(furan-2-yl)but-3-enylamino)phenol (10): Purified by flash chromatography (5-20\% EtOAc/hex) to afford the title compound as a yellow oil (162 mg, 71\%). ${ }^{1} \mathrm{H} \mathrm{NMR}\left(300 \mathrm{MHz}, \mathrm{CDCl}_{3}\right) \delta$ $7.39(\mathrm{dd}, J=1.8,0.7 \mathrm{~Hz}, 1 \mathrm{H}$, Fur-H), 6.85-6.65 (m, 4H, Ar-H), 6.32 (dd, $J=3.2,1.8 \mathrm{~Hz}, 1 \mathrm{H}$, Fur-H), 6.20 $\left(\mathrm{dt}, J=3.2,0.7 \mathrm{~Hz}, 1 \mathrm{H}\right.$, Fur-H) $5.91-5.76\left(\mathrm{~m}, 1 \mathrm{H}, \mathrm{CH}_{2}-\mathrm{CH}=\mathrm{CH}_{2}\right), 5.27-5.14\left(\mathrm{~m}, 2 \mathrm{H}, \mathrm{CH}_{2}-\mathrm{CH}=\mathrm{CH}_{2}\right)$, 5.50-4.00 (br s, 1H, N-H or O-H), 4.52 (br t, $J=6.5 \mathrm{~Hz}, 1 \mathrm{H}$ ), 2.73 (apparent t, $J=6.8 \mathrm{~Hz}, 2 \mathrm{H}, \mathrm{CH}_{2^{-}}$ $\left.\mathrm{CH}=\mathrm{CH}_{2}\right) ;{ }^{13} \mathrm{C} \mathrm{NMR}\left(75 \mathrm{MHz}, \mathrm{CDCl}_{3}\right) \delta 155.7,144.8,141.6,135.5,134.1,121.3,119.1,118.5,114.8$, 114.6, 110.2, 106.4, 52.4, 39.3; IR (thin film): 3600-2500 (br s), 3077 (m), 1640 (w), 1610 (s), 1512 (s),

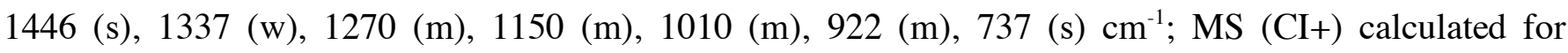
$\mathrm{C}_{14} \mathrm{H}_{15} \mathrm{NO}_{2}$ : 229, observed: $230(\mathrm{M}+1) ;[\alpha]_{\mathrm{D}}=+77.0^{\circ}\left(\right.$ c $\left.0.78, \mathrm{CHCl}_{3}\right)$.

2-((1R,2R)-2-methyl-1-phenylbut-3-enylamino)phenol (11): Reaction of the imine derived from benzaldehyde and 2-aminophenol with $(S, S)-\mathbf{4}$ followed by workup as in the general procedure, and flash chromatography (10-15-20\% EtOAc/hex) gave $161 \mathrm{mg}(64 \%)$ of the title compound as an off-white solid.

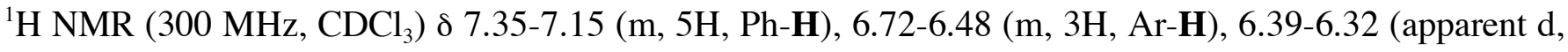
$1 \mathrm{H}, \mathrm{Ar}-\mathbf{H}), 5.85-5.71\left(\mathrm{~m}, 1 \mathrm{H}, \mathrm{CH}_{2}-\mathrm{CH}=\mathrm{CH}_{2}\right), 5.23-5.12\left(\mathrm{~m}, 2 \mathrm{H}, \mathrm{CH}_{2}-\mathrm{CH}=\mathrm{CH}_{2}\right), 4.94-4.63$ (br s, 1H, N-H or O-H), 4.63-4.25 (br s, 1H, N-H or O-H), 4.04 (d, J = 6.9Hz, 1H, Ph-CH-NH), 2.64-2.50 (m, 1H, CH$\left.\left.\mathrm{CH}=\mathrm{CH}_{2}\right), 1.00(\mathrm{~d}, 3 \mathrm{H}, J=6.9 \mathrm{~Hz}) \mathrm{Ph}(\mathrm{NHAr}) \mathrm{CHCH}\left(\mathrm{CH}_{3}\right) \mathrm{CH}=\mathrm{CH}_{2}\right) ;{ }^{13} \mathrm{C} \mathrm{NMR}\left(75 \mathrm{MHz}, \mathrm{CDCl}_{3}\right) \delta 144.3$, 143.0, 142.1, 136.8, 128.8, 127.9, 127.6, 121.8, 118.1, 116.8, 114.8, 114.2, 63.5, 45.7, 17.9; IR (thin film) 3600-3300 (br s), 3395 (m), 2981 (m), 1640 (m), 1607 (s), 1591 (s), 1479 (s), 1446 (s), 1319 (s), 1266 (s), 1154 (s), 1136 (s), 1097 (s), 908 (s), 732 (s), 702 (s), 680 (s) cm-1; MS (CI+) calculated for $\mathrm{C}_{17} \mathrm{H}_{19} \mathrm{NO}^{-}$ 253, observed: $254(\mathrm{M}+1) ;[\alpha]_{\mathrm{D}}=+13.6^{\circ}\left(c 1.30, \mathrm{CHCl}_{3}\right)$.

2-((1R,2S)-2-methyl-1-phenylbut-3-enylamino)phenol (12): Reaction of the imine derived from benzaldehyde and 2-aminophenol with $(S, S)-5$ followed by workup as in the general procedure, and flash chromatography (10-15-20\% EtOAc/hex) gave $186 \mathrm{mg}$ (74\%) of the title compound as an off-white solid. ${ }^{1} \mathrm{H}$ NMR (300 MHz, $\mathrm{CDCl}_{3}$ ) 8 7.29-7.15 (m, 5H, Ph-H), 6.80-6.25 (br m, 4H, Ar-H), 6.39-6.32 (apparent d, $1 \mathrm{H}, \mathrm{Ar}-\mathbf{H}), 5.80-5.67\left(\mathrm{~m}, 1 \mathrm{H}, \mathrm{CH}_{2}-\mathrm{CH}=\mathrm{CH}_{2}\right), 5.17-5.06\left(\mathrm{~m}, 2 \mathrm{H}, \mathrm{CH}_{2}-\mathrm{CH}=\mathrm{CH}_{2}\right), 5.00-4.15(2 \mathrm{br} \mathrm{s}, 3 \mathrm{H}$, $\mathrm{N}-\mathbf{H}, \quad \mathrm{O}-\mathrm{H}, \quad$ and $\mathrm{Ph}-\mathrm{CH}-\mathrm{NH}), \quad 2.76-2.64\left(\mathrm{~m}, \quad 1 \mathrm{H}, \quad \mathrm{CH}-\mathrm{CH}=\mathrm{CH}_{2}\right), 1.03(\mathrm{~d}, \quad 3 \mathrm{H}, \quad J=7.0 \mathrm{~Hz})$ 
$\left.\mathrm{Ph}(\mathrm{NHAr}) \mathrm{CHCH}\left(\mathrm{CH}_{3}\right) \mathrm{CH}=\mathrm{CH}_{2}\right) ;{ }^{13} \mathrm{C} \mathrm{NMR}\left(75 \mathrm{MHz}, \mathrm{CDCl}_{3}\right) \delta 143.3,141.1,140.3,136.2,128.2,127.6$, 127.0, 121.6, 117.3, 116.0, 114.4, 113.4, 61.8, 44.0, 15.7; IR (thin film) 3600-2800 (br s), 3423 (m), 3061 (m), 2966 (m), 1640 (w), 1611 (s), 1513 (s), 1452 (s), 1264 (m), 1202 (m), 1096 (m), 918 (m), 739 (s), $702 \mathrm{~cm}^{-1}$; MS (CI+) calculated for $\mathrm{C}_{17} \mathrm{H}_{19} \mathrm{NO}$ : 253, observed: $254(\mathrm{M}+1) ;[\alpha]_{\mathrm{D}}=-13^{\circ}\left(c 1.31, \mathrm{CHCl}_{3}\right)$.

Preparation of $N$-allyl and $N$-homoallyl phenolimines (14 and 16): The requisite amine (5.5 mmol) is added to 2'-hydroxyacetophenone ( $5 \mathrm{mmol}$, neat) and the mixture is stirred at ambient temperature for 15 min. The reaction mixture is diluted with EtOAc, washed with brine, dried over $\mathrm{Na}_{2} \mathrm{SO}_{4}$ and concentrated. Purification of the residue by flash chromatography on silica gel affords the pure imine products.

Representative procedure for the enantioselective allylation of phenolimines with $(S, S)-1$ (Scheme 3): To a solution of the imine $(5.0 \mathrm{mmol})$ in PhMe $(5 \mathrm{~mL})$ is added $(S, S)-1(7.5 \mathrm{mmol})$ and the resulting mixture is heated to reflux and stirred for $6 \mathrm{~h}$. The reaction is quenched by the addition of $1.25 \mathrm{~N}$ $\mathrm{HCl}$ in $\mathrm{MeOH}(0.5 \mathrm{~mL})$. The resulting mixture is stirred for $15 \mathrm{~min}$, and then concentrated. The residue is then treated with EtOAc $(25 \mathrm{~mL})$ and $3 \mathrm{~N} \mathrm{NaOH}(25 \mathrm{~mL})$. The layers are separated and the aqueous layer is extracted with EtOAc $(2 \times 10 \mathrm{~mL})$. The combined organic layers are washed with brine $(10 \mathrm{~mL})$, dried over $\mathrm{Na}_{2} \mathrm{SO}_{4}$, filtered, and concentrated. Purification of the residue by flash chromatography on silica gel affords the pure amine products in the yields and enantioselectivities reported.

Representative procedure for the one-pot enantioselective allylation/RCM using $(S, S)-1$ and Grubbs-II (Scheme 4): The allylation is carried out as described in the above procedure except that instead of quenching the reaction mixture with $\mathrm{HCl} / \mathrm{MeOH}$, the Grubbs second-generation catalyst is added $(0.025 \mathrm{mmol})$ and the resulting mixture is heated to $40{ }^{\circ} \mathrm{C}$ and stirred for $16 \mathrm{~h}$. The reaction is then quenched by the addition of $1.25 \mathrm{~N} \mathrm{HCl}$ in $\mathrm{MeOH}(0.5 \mathrm{~mL})$ and the work-up carried out as described in the above procedure.

(S)-2-(1-Allylamino-1-methyl-but-3-enyl)-phenol (15): Prepared utilizing the general allylation procedure described above. Purified by flash chromatography (10\% EtOAc/hex) to afford the title compound as a clear oil (101 mg, 93\%). ${ }^{1} \mathrm{H}$ NMR (300 MHz, $\mathrm{CDCl}_{3}$ ) $\delta$ 7.21-7.12 (1H, Ar-H), 7.08-6.96 $(1 \mathrm{H}, \operatorname{Ar}-\mathbf{H}), \quad 6.85-6.72(\mathrm{~m}, 2 \mathrm{H}, \mathrm{Ar}-\mathbf{H}), \quad 6.00-5.84\left(\mathrm{~m}, 1 \mathrm{H}, \quad \mathrm{NCH}_{2} \mathrm{CH}=\mathrm{CH}_{2}\right), \quad 5.80-5.62(\mathrm{~m}, 1 \mathrm{H}$, $\left.\mathrm{CCH}_{2} \mathrm{CH}=\mathrm{CH}_{2}\right), \quad 5.25-5.06 \quad\left(\mathrm{~m}, \quad 4 \mathrm{H}, \quad \mathrm{NCH}_{2} \mathrm{CH}=\mathrm{CH}_{2}\right.$ and $\left.\mathrm{CCH}_{2} \mathrm{CH}=\mathrm{CH}_{2}\right), \quad 3.23-3.00 \quad(\mathrm{~m}, \quad 2 \mathrm{H}$, $\left.\mathrm{NCH}_{2} \mathrm{CH}=\mathrm{CH}_{2}\right), 2.65-2.54\left(\mathrm{~m}, 2 \mathrm{H}, \mathrm{CCH}_{2} \mathrm{CH}=\mathrm{CH}_{2}\right), 1.60-1.50(\mathrm{~s}, 3 \mathrm{H}, \mathrm{Me}) ;{ }^{13} \mathrm{C} \mathrm{NMR}\left(75 \mathrm{MHz}, \mathrm{CDCl}_{3}\right) \delta$ $157.2,135.4,132.8,128.7,128.0,126.8,120.0,118.7,116.9,116.5,59.7,44.8,24.0$; IR (thin film) 3299, 
3077, 2978, 2921, 2858, 2732, 2631, 1642, 1609, 1583, 1494, 1467, 1407, 1253, 1160, $921 \mathrm{~cm}^{-1}$; LRMS $(\mathrm{FAB}+)$ calculated for $\mathrm{C}_{14} \mathrm{H}_{19} \mathrm{NO}: 217$, found: $218(\mathrm{M}+\mathrm{H}) ;[\alpha]_{\mathrm{D}}=+58^{\circ}\left(c 1.00, \mathrm{CHCl}_{3}\right)$.

(S)-2-(1-But-3-enylamino-1-methyl-but-3-enyl)-phenol (17): Prepared utilizing the general allylation procedure described above. Purified by flash chromatography (10\%EtOAc/hex) to afford the title compound as a clear oil (104 mg, 90\%). ${ }^{1} \mathrm{H}$ NMR $\left(300 \mathrm{MHz}, \mathrm{CDCl}_{3}\right) \delta$ 7.20-7.10 (t, 1H, Ar-H), 7.06-6.98 (d, 1H, Ar-H), 6.84-6.74 (m, 2H, Ar-H), 5.82-5.60 (m, 2H, $\mathrm{NCH}_{2} \mathrm{CH}_{2} \mathrm{CH}_{\mathbf{H}} \mathrm{CH}_{2}$ and $\left.\mathrm{CCH}_{2} \mathrm{CH}=\mathrm{CH}_{2}\right), \quad 5.22-5.02\left(\mathrm{~m}, 4 \mathrm{H}, \quad \mathrm{NCH}_{2} \mathrm{CH}_{2} \mathrm{CH}=\mathrm{CH}_{2}\right.$ and $\left.\mathrm{CCH}_{2} \mathrm{CH}=\mathrm{CH}_{2}\right), \quad 2.85-2.40 \quad(\mathrm{~m}, 4 \mathrm{H}$, $\mathrm{NCH}_{2} \mathrm{CH}_{2} \mathrm{CH}=\mathrm{CH}_{2}$ and $\left.\mathrm{CCH}_{2} \mathrm{CH}=\mathrm{CH}_{2}\right), 2.39-2.16\left(\mathrm{~m}, 2 \mathrm{H}, \mathrm{NCH}_{2} \mathrm{CH}_{2} \mathrm{CH}=\mathrm{CH}_{2}\right), 1.55-1.45$ (s, 3H, Me); ${ }^{13} \mathrm{C}$ NMR $\left(75 \mathrm{MHz}, \mathrm{CDCl}_{3}\right) \delta 157.7,135.5,132.8,128.7,128.2,126.7,119.9,118.6,117.3,116.9,59.5$, 40.6, 34.2, 23.9; IR (thin film) 3300, 3077, 2978, 2926, 1640, 1609, 1584, 1496, 1466, 1412, 1254, 995 $\mathrm{cm}^{-1}$; LRMS (FAB+) calculated for $\mathrm{C}_{15} \mathrm{H}_{21} \mathrm{NO}: 231$, found: $232(\mathrm{M}+\mathrm{H}) ;[\alpha]_{\mathrm{D}}=+36^{\circ}\left(c 0.50, \mathrm{CHCl}_{3}\right)$.

(S,R)-2-(1-Allylamino-1,2-dimethyl-but-3-enyl)-phenol (18): Prepared utilizing the general crotylation procedure described above with $(S, S)-5$ except that the mixture was reacted for $48 \mathrm{~h}$ instead of 6h. Purified by flash chromatography (10\% EtOAc/hex) to afford the title compound as a clear oil (73 mg, 63\%). ${ }^{1} \mathrm{H}$ NMR (300 MHz, $\mathrm{CDCl}_{3}$ ) $\delta$ 7.20-7.10 (m, 1H, Ar-H), 6.99-6.91 (m, 1H, Ar-H), 6.87-6.79 (m, $1 \mathrm{H}, \operatorname{Ar}-\mathbf{H}), \quad 6.79-6.72(\mathrm{~m}, 1 \mathrm{H}, \mathrm{Ar}-\mathbf{H}), 6.01-5.86\left(\mathrm{~m}, 1 \mathrm{H}, \quad \mathrm{NCH}_{2} \mathrm{CH}=\mathrm{CH}_{2}\right), \quad 5.70-5.52(\mathrm{~m}, 1 \mathrm{H}$, $\left.\mathrm{CCHMeCH}=\mathrm{CH}_{2}\right), 5.30-5.10\left(\mathrm{~m}, 2 \mathrm{H}, \mathrm{NCH}_{2} \mathrm{CH}=\mathrm{CH}_{2}\right), 5.00-4.85\left(\mathrm{~m}, 2 \mathrm{H}, \mathrm{CCHMeCH}=\mathrm{CH}_{2}\right), 3.37-3.21$ $\left(\mathrm{m}, 1 \mathrm{H}, \mathrm{NCH}_{2} \mathrm{CH}=\mathrm{CH}_{2}\right), 3.10-2.93\left(\mathrm{~m}, 1 \mathrm{H}, \mathrm{NCH}_{2} \mathrm{CH}=\mathrm{CH}_{2}\right), 2.83-2.65\left(\mathrm{~m}, 1 \mathrm{H}, \mathrm{CCHMeCH}=\mathrm{CH}_{2}\right), 1.55-$ $1.42(\mathrm{~s}, 3 \mathrm{H}, \mathrm{Me}), 1.11-1.04\left(\mathrm{~d}, 3 \mathrm{H}, \mathrm{J}=6.89 \mathrm{~Hz}, \mathrm{CCHMeCH}=\mathrm{CH}_{2}\right) ;{ }^{13} \mathrm{C} \mathrm{NMR}\left(75 \mathrm{MHz}, \mathrm{CDCl}_{3}\right) \delta 156.8$, $139.4,135.3,128.7,128.5,118.4,116.8,115.8,63.2,46.7,45.1,29.7,20.8,13.9$; IR (thin film) 3313, 3080, 2976, 2926, 2856, 1608, 1583, 1493, 1464, 1409, 1251, 1118, $919 \mathrm{~cm}^{-1}$; LRMS (FAB+) calculated for $\mathrm{C}_{15} \mathrm{H}_{21} \mathrm{NO}$ : 231, found: $232(\mathrm{M}+\mathrm{H}) ;[\alpha]_{\mathrm{D}}=-98^{\circ}\left(c 1.00, \mathrm{CHCl}_{3}\right)$.

(S)-2-(2-Methyl-1,2,3,6-tetrahydro-pyridin-2-yl)-phenol (19): Prepared utilizing the general allylation/RCM procedure described above. Purified by flash chromatography (20\%EtOAc/hex) to afford the title compound as a clear oil (85 mg, 90\%). ${ }^{1} \mathrm{H}$ NMR (300 MHz, $\left.\mathrm{CDCl}_{3}\right) \delta$ 7.20-7.12 (1H, Ar-H), 7.10-7.15 (1H, Ar-H), 6.90-6.85 (1H, Ar-H), 5.98-5.80 (1H, CH=CH), 5.75-5.60 (1H, CH=CH), 3.55$3.36\left(1 \mathrm{H}, \quad \mathrm{NHCH}_{2}\right), \quad 3.32-3.18\left(1 \mathrm{H}, \quad \mathrm{NHCH}_{2}\right), \quad 2.90-2.70 \quad\left(1 \mathrm{H}, \quad \mathrm{CCH}_{2} \mathrm{CH}=\mathrm{CH}\right), \quad 2.35-2.18 \quad(1 \mathrm{H}$, $\left.\mathrm{CCH}_{2} \mathrm{CH}=\mathrm{CH}\right), 1.60-1.45(3 \mathrm{H}, \mathrm{Me}) ;{ }^{13} \mathrm{C} \mathrm{NMR}\left(75 \mathrm{MHz}, \mathrm{CDCl}_{3}\right) \delta 157.8,128.7,125.7,125.2,124.0$, 118.8, 117.2, 55.5, 40.9, 34.6, 25.9; IR (thin film) 3413, 2919, 1643, 1467, 1256, $752 \mathrm{~cm}^{-1}$; LRMS $(\mathrm{FAB}+)$ calculated for $\mathrm{C}_{12} \mathrm{H}_{15} \mathrm{NO}: 189$, found: $190(\mathrm{M}+\mathrm{H}) ;[\alpha]_{\mathrm{D}}=+57^{\circ}\left(c 0.50, \mathrm{CHCl}_{3}\right)$. 
(S)-2-(2-Methyl-2,3,6,7-tetrahydro-1H-azepin-2-yl)-phenol (20): Prepared utilizing the general allylation/RCM procedure described above. Purified by flash chromatography (20\% EtOAc/hex) to afford the title compound as a clear oil (83 mg, 82\%). ${ }^{1} \mathrm{H}$ NMR $\left(300 \mathrm{MHz}, \mathrm{CDCl}_{3}\right) \delta$ 7.20-7.12 (t, $\left.1 \mathrm{H}, \mathrm{Ar}-\mathbf{H}\right)$, 7.08-7.02 (d, 1H, Ar-H), 6.84-6.74 (m, 2H, Ar-H), 5.90-5.75 (m, 2H, CH=CH), 3.06-2.90 $\left(\mathrm{NCH}_{2} \mathrm{CH}_{2} \mathrm{CH}=\mathrm{CH}_{2}\right), 2.83-2.70\left(\mathrm{~m}, 1 \mathrm{H}, \mathrm{CCH}_{2} \mathrm{CH}=\mathrm{CH}_{2}\right), 2.65-2.54\left(\mathrm{~m}, 1 \mathrm{H}, \mathrm{CCH}_{2} \mathrm{CH}=\mathrm{CH}_{2}\right), 2.38-2.25$ $\left(\mathrm{m}, 2 \mathrm{H}, \mathrm{NCH}_{2} \mathrm{CH}_{2} \mathrm{CH}=\mathrm{CH}_{2}\right), 1.45-1.40(\mathrm{~s}, 3 \mathrm{H}, \mathrm{Me}) ;{ }^{13} \mathrm{C} \mathrm{NMR}\left(75 \mathrm{MHz}, \mathrm{CDCl}_{3}\right) \delta 158.08,131.4,128.5$, 128.4, 127.6, 127.1, 118.5, 117.0, 60.4, 40.8, 39.7, 31.0, 29.9; IR (thin film) 3306, 2926, 2360, 1585, 1495, 1463, 1415, 1256, $753 \mathrm{~cm}^{-1}$; LRMS (FAB+) calculated for $\mathrm{C}_{13} \mathrm{H}_{17} \mathrm{NO}: 203$, found: $204(\mathrm{M}+\mathrm{H}) ;[\alpha]_{\mathrm{D}}$ $=-27^{\circ}\left(c 1.50, \mathrm{CHCl}_{3}\right)$.

\section{Stereochemical Proofs.}

For the allylation and crotylation reactions described in Scheme 2, the relative and absolute configurations were established as follows. Compound $\mathbf{1 1}$ was converted into the known compound $\mathbf{2 1}^{1}$ by the sequence of reactions shown in Scheme 5. Comparison of spectral data confirmed the assignment of relative configuration, and comparison of the optical rotation allowed the assignment of absolute configuration. The absolute configuration of all other products in Scheme 2, and the relative configuration of 12 and 13 were assigned by analogy.

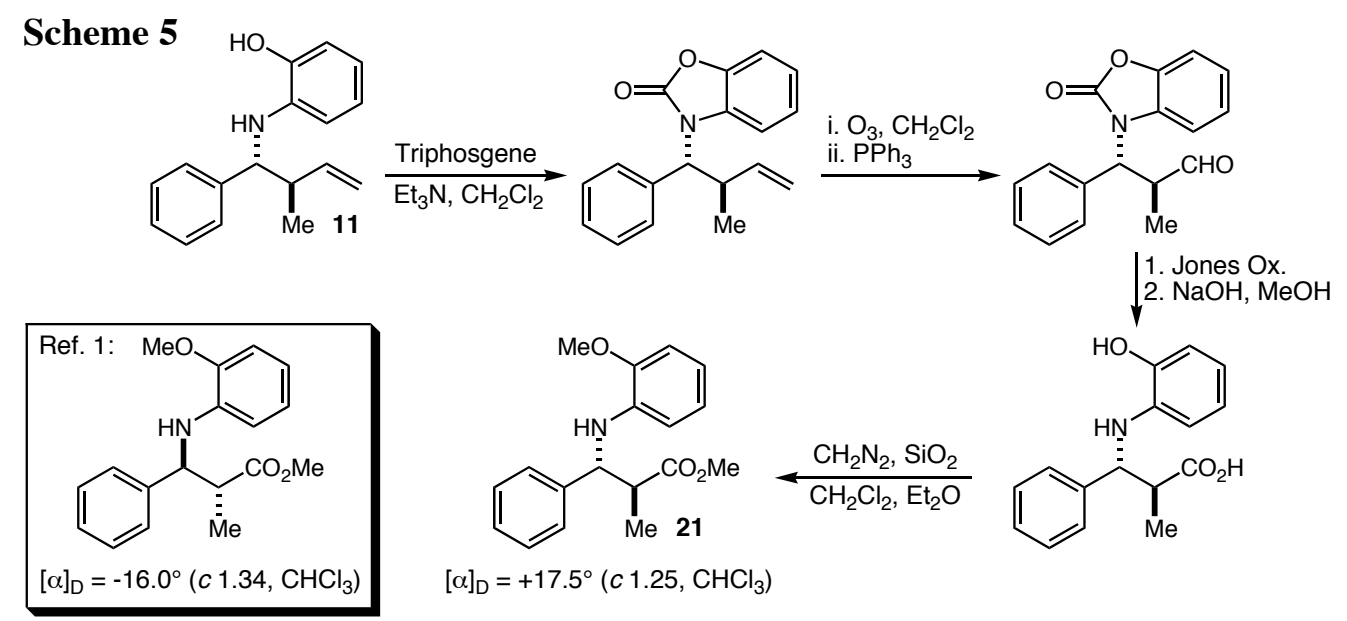

The absolute configuration of amine $\mathbf{1 5}$ was established as follows. Our previously reported acylhydrazone allylation chemistry ${ }^{2}$ was employed to provide an authentic sample of $(R)-\mathbf{1 5}$ as described in Scheme 6. Comparison of the optical rotation to that of amine 15 from Scheme 3 in the present work allowed the assignment of absolute configuration. The absolute configuration of amines $\mathbf{1 7}$ and $\mathbf{1 8}$ was assigned by analogy. 


\section{Scheme 6}<smiles>C=CC[Si]1(Cl)O[C@H](c2ccccc2)[C@H](C)[C@H]1N(C)SC</smiles>

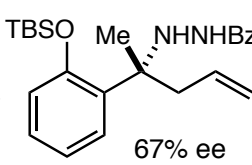

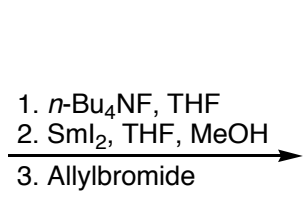

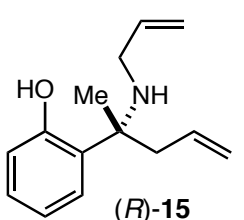
$[\alpha]_{D}=-35^{\circ}\left(c 0.50, \mathrm{CHCl}_{3}\right)$

$(67 \%$ ee)

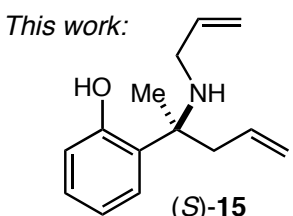

$[\alpha]_{D}=+58^{\circ}\left(c 1.0, \mathrm{CHCl}_{3}\right)$ $(98 \%$ ee)

The relative configuration of amine 18 was established by subjecting $\mathbf{1 8}$ to a ring closing metathesis reaction with the Grubbs second-generation catalyst to give 22 (Scheme 7). The illustrated selective 1D NOESY enhancements were observed and established the relative stereochemistry of $\mathbf{1 8}$ as shown.

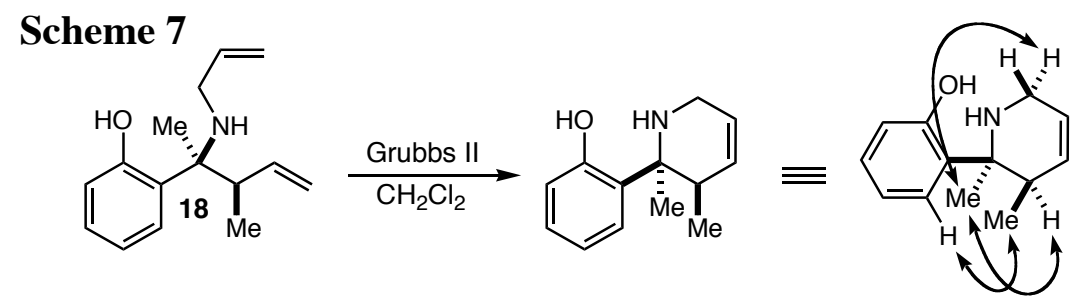

Determination of Enantiomeric Excess (ee): All ees were determined by HPLC analysis with a chiral stationary phase using the conditions noted below. NOTE: In many cases the most convenient method for the preparation of authentic racemic samples was to admix equimolar amounts of the $(S, S)$ and $(R, R)$ antipodes of the requisite silane $(\mathbf{1}, \mathbf{4}$, and $\mathbf{5})$. This procedure entails greater error, and we attribute the deviation from 50:50 in some of our racemic HPLC traces to this phenomenon.

3. Chiralcel OJ-H, $5 \%$ - $\mathrm{PrOH} /$ hexane, $1.0 \mathrm{~mL} / \mathrm{min}, 254 \mathrm{~nm}$ :
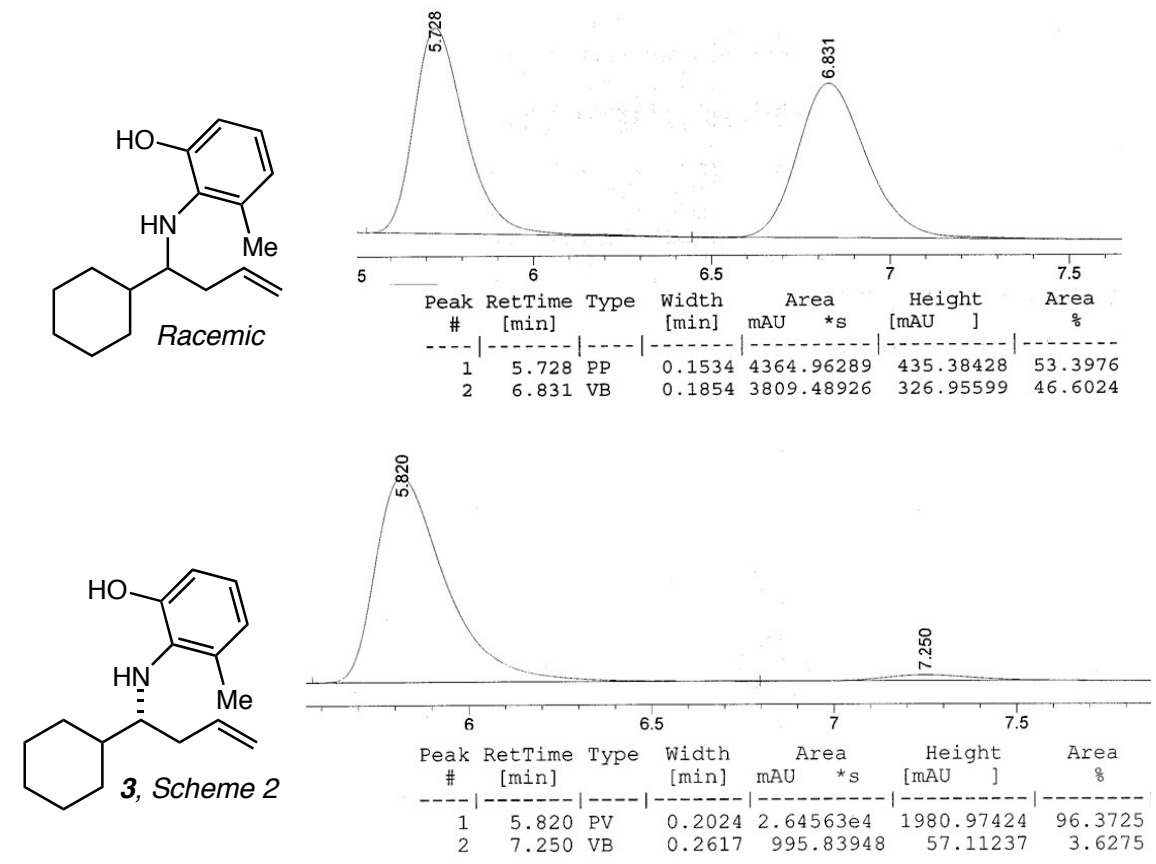
6. Chiralcel OJ-H, $10 \% \mathrm{EtOH} /$ hexane, $1.0 \mathrm{~mL} / \mathrm{min}, 254 \mathrm{~nm}$ :
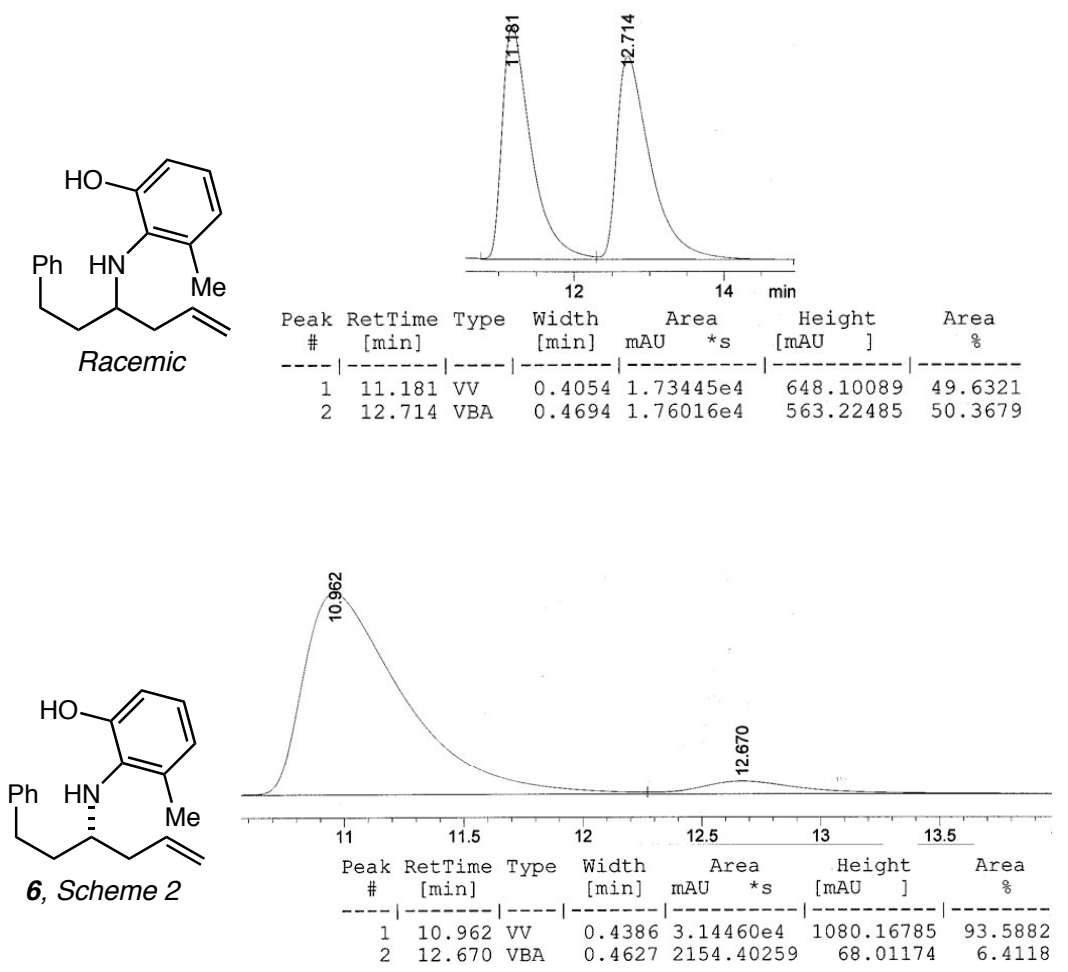

8. Chiralpak AD-H, $25 \%$ i-PrOH/hexane, $1.0 \mathrm{~mL} / \mathrm{min}, 220 \mathrm{~nm}$ :
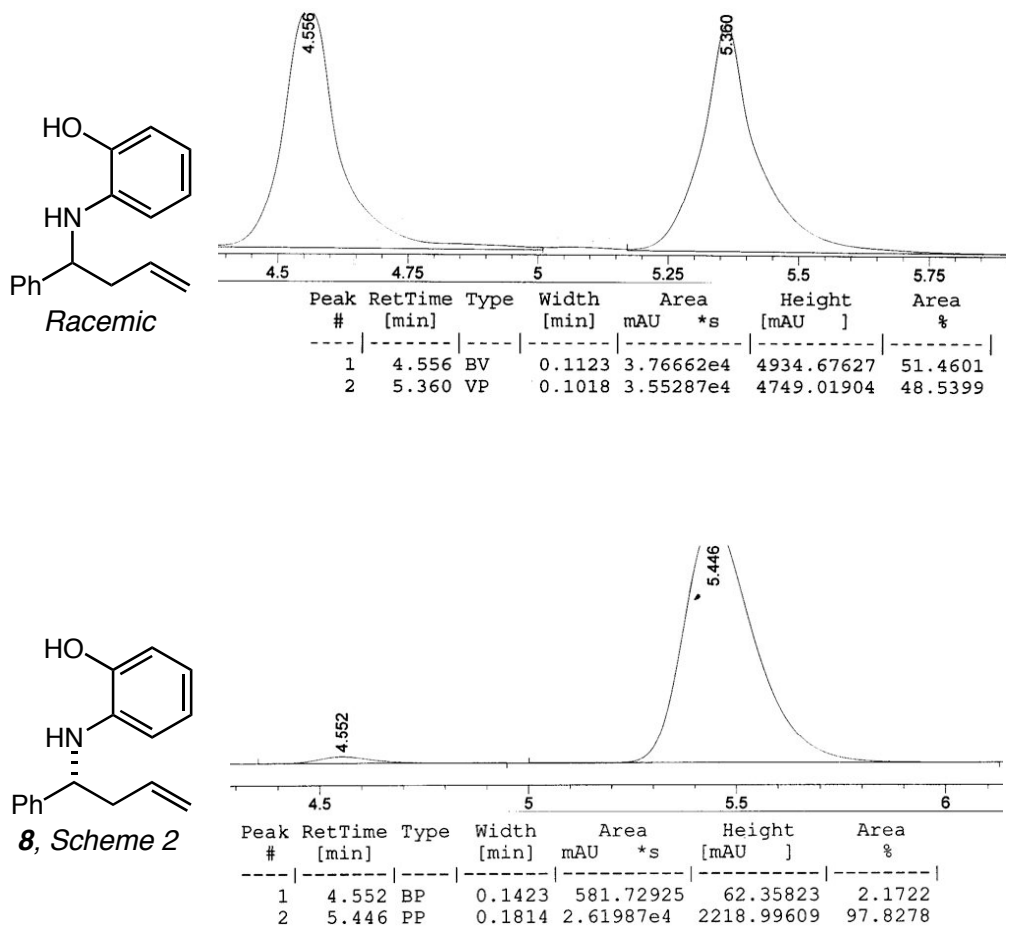
9. Chiralpak AD-H, 20\% i-PrOH/hexane, $1.0 \mathrm{~mL} / \mathrm{min}, 254 \mathrm{~nm}$ :
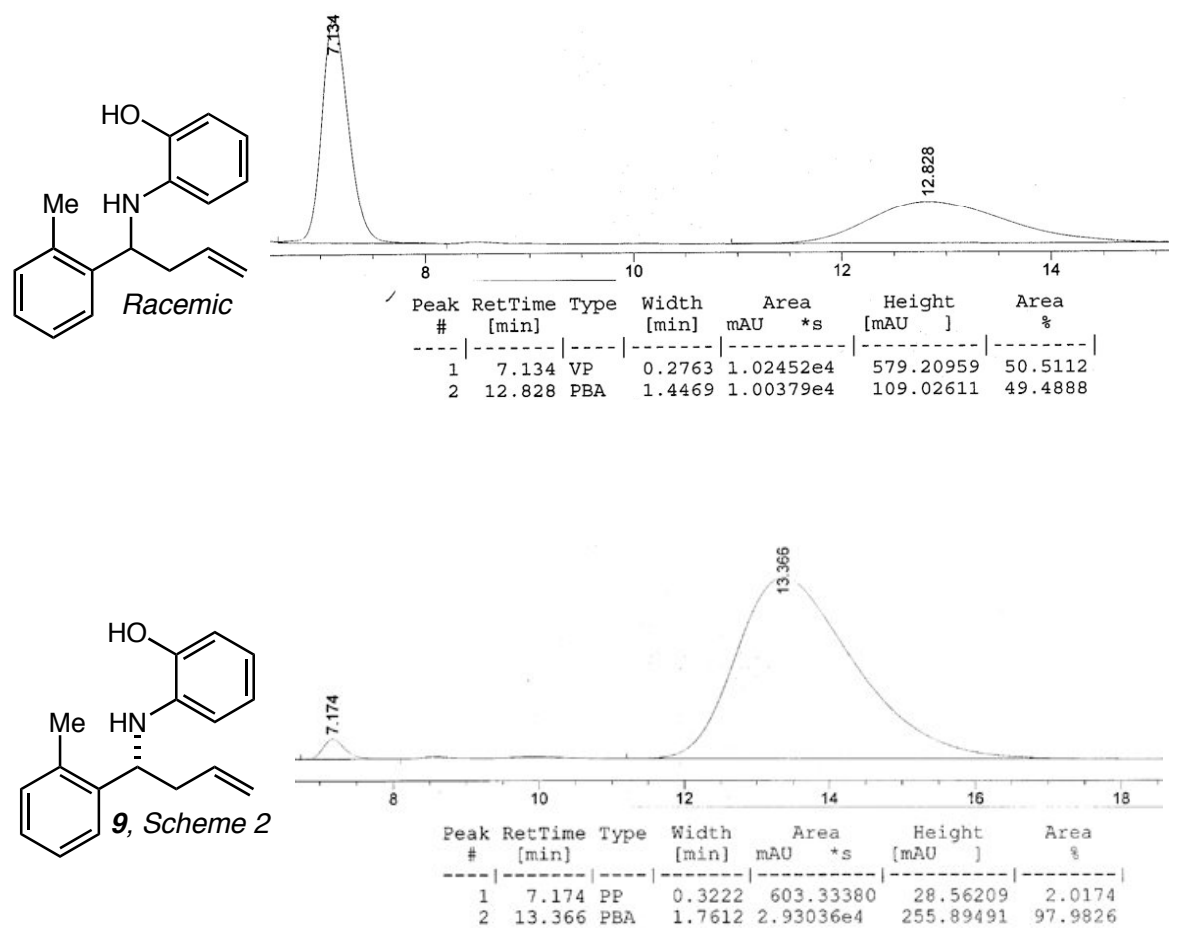

10. Chiralpak AD-H, $10 \% i$-PrOH/hexane, $1.0 \mathrm{~mL} / \mathrm{min}, 220 \mathrm{~nm}$ :
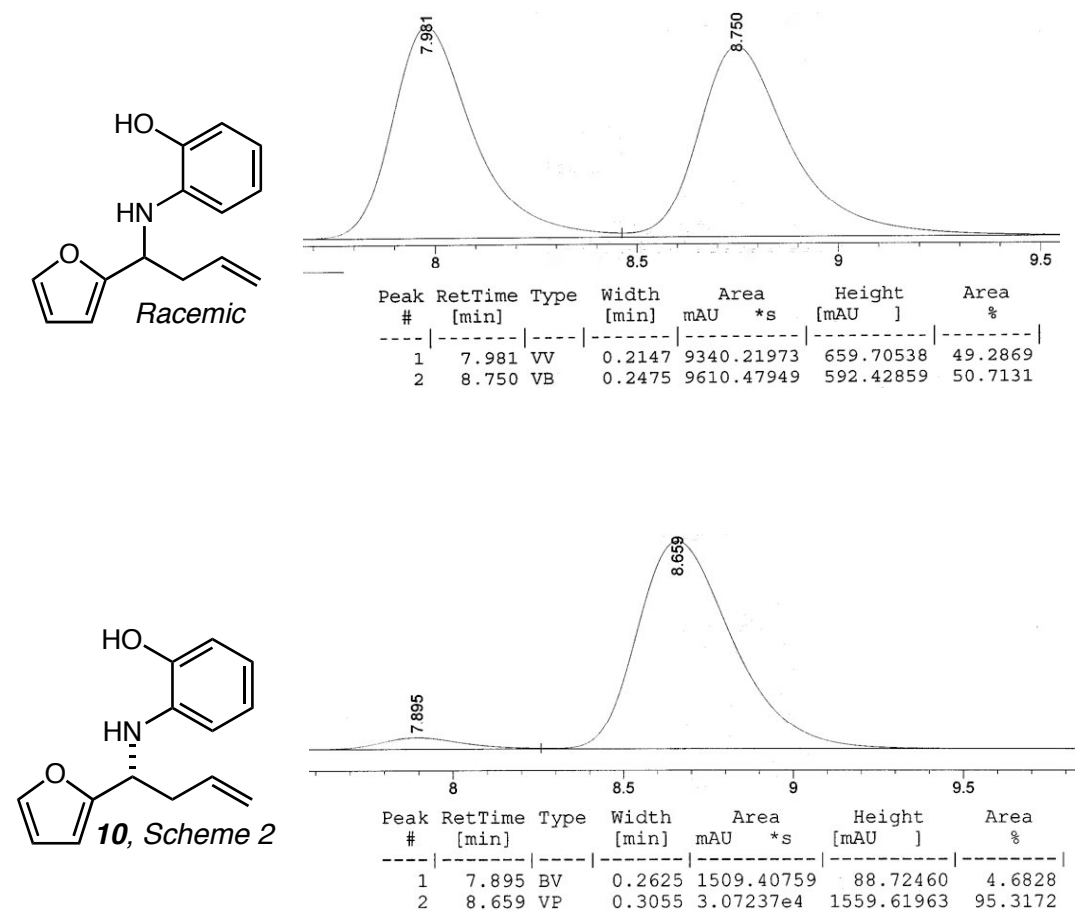
11. Chiralpak AD-H, $10 \% i$-PrOH/hexane, $1.0 \mathrm{~mL} / \mathrm{min}, 254 \mathrm{~nm}$ :
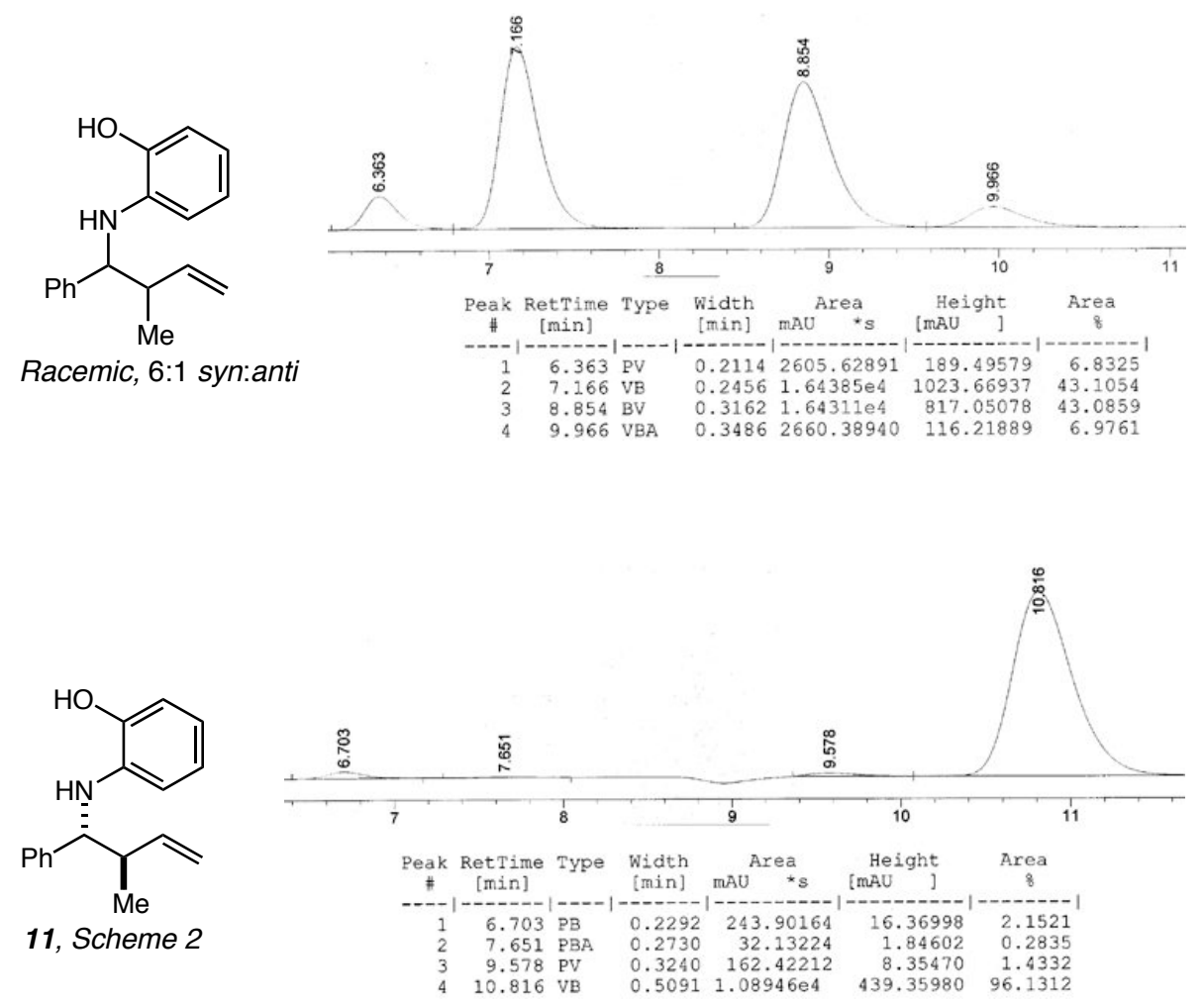

12. Chiralpak AD-H, $10 \% i$-PrOH/hexane, $1.0 \mathrm{~mL} / \mathrm{min}, 254 \mathrm{~nm}$ :

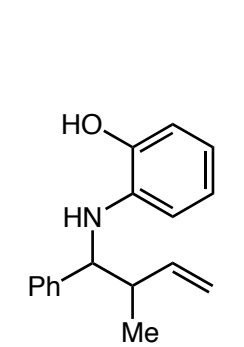

Racemic, 6:1 syn:anti
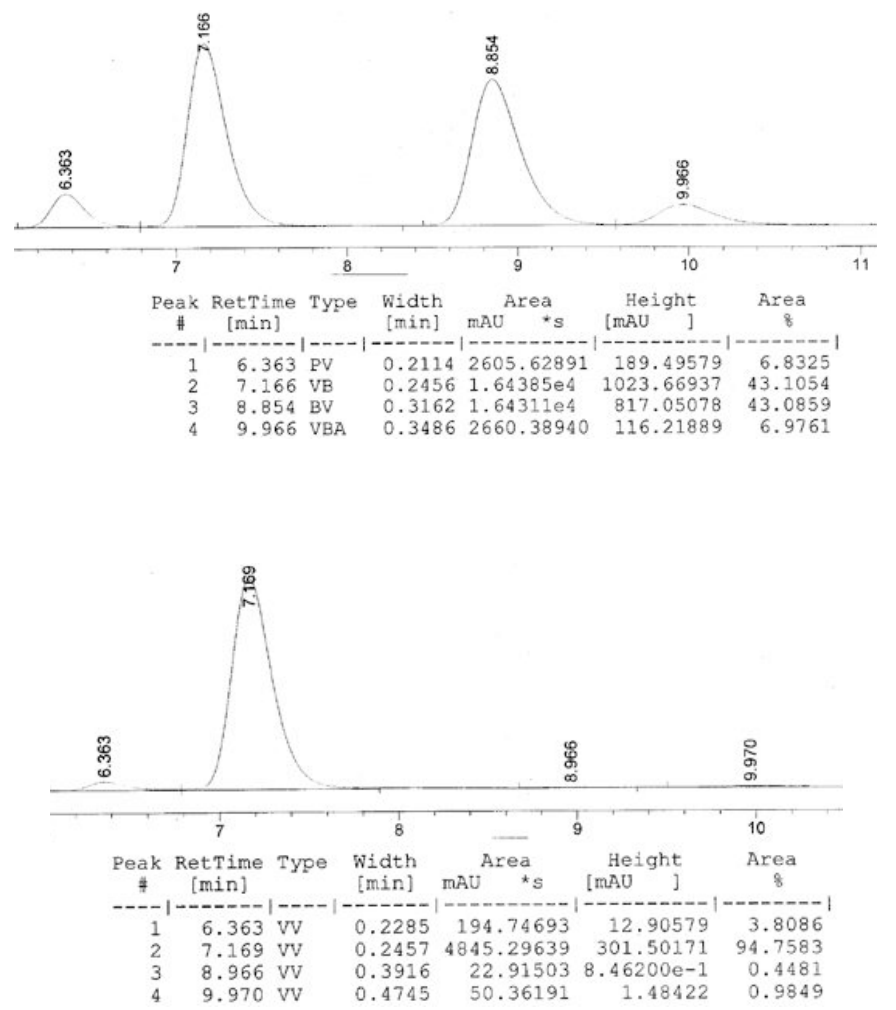
13. Chiralcel OJ-H, 4\% i-PrOH/hexane, $1.0 \mathrm{~mL} / \mathrm{min}, 254 \mathrm{~nm}$ :
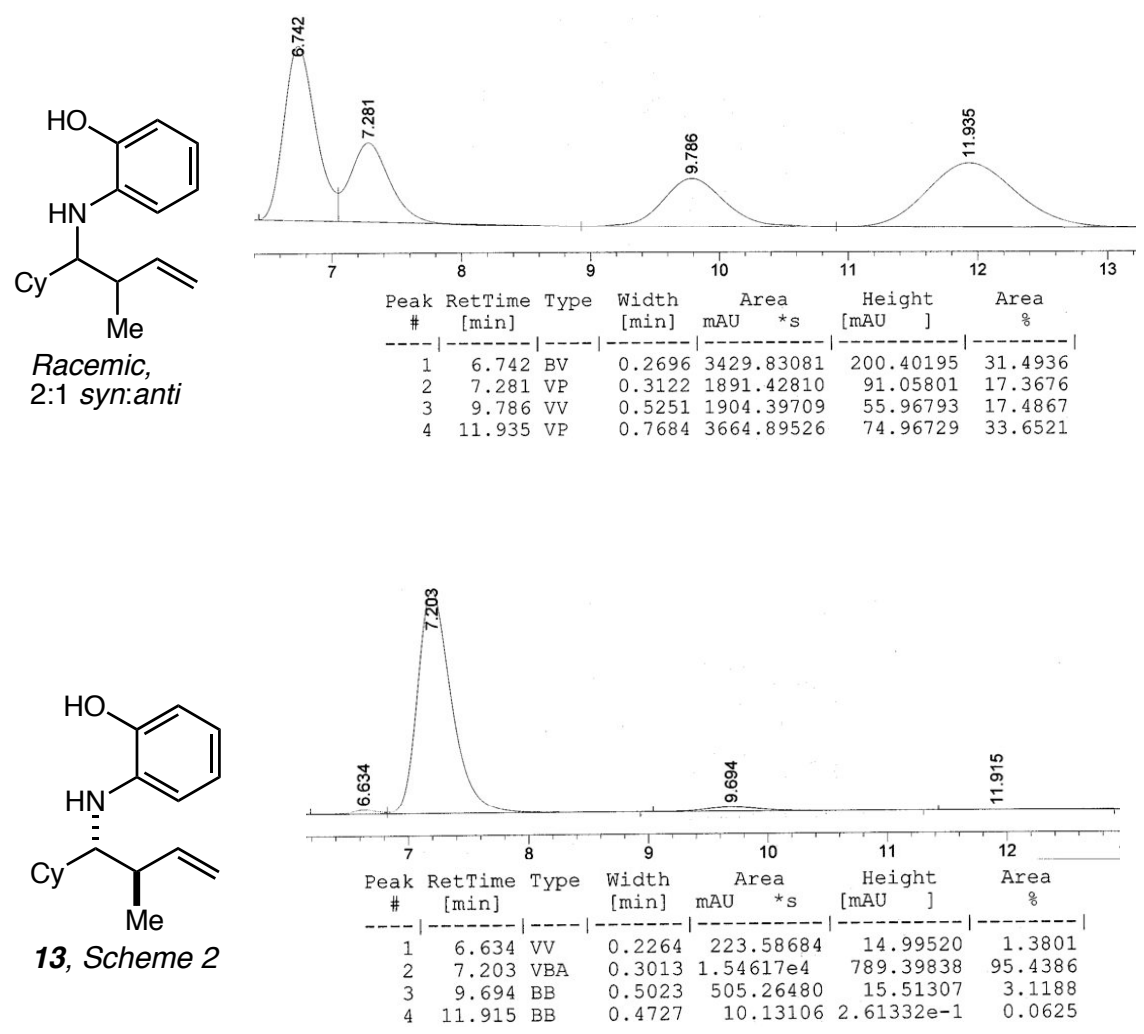

15. Chiralpak AD-H, 1\% EtOH/hexane, $1.0 \mathrm{~mL} / \mathrm{min}, 220 \mathrm{~nm}$ :

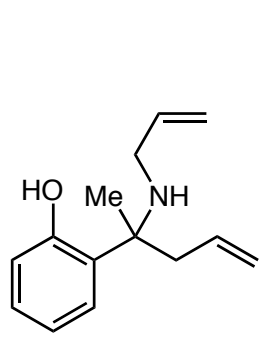

Racemic

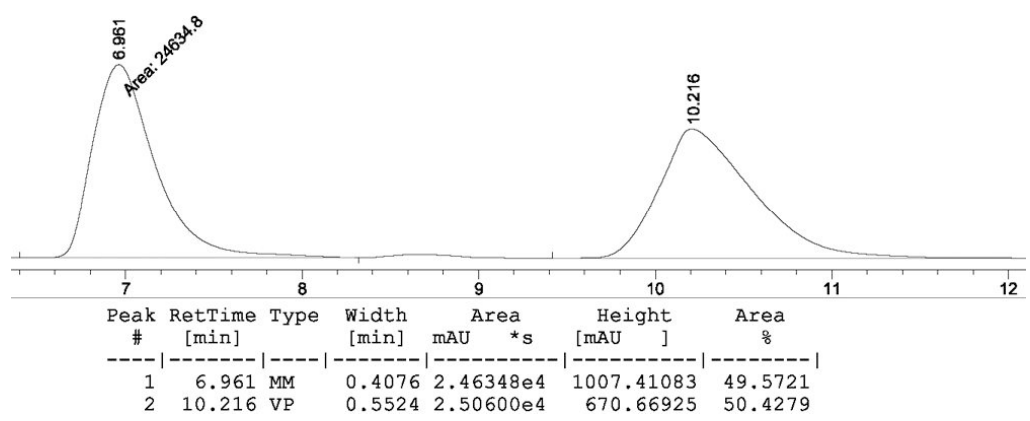

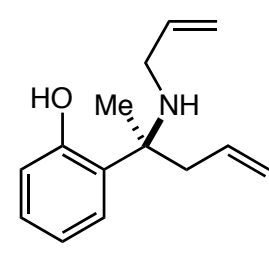

15, Scheme 3

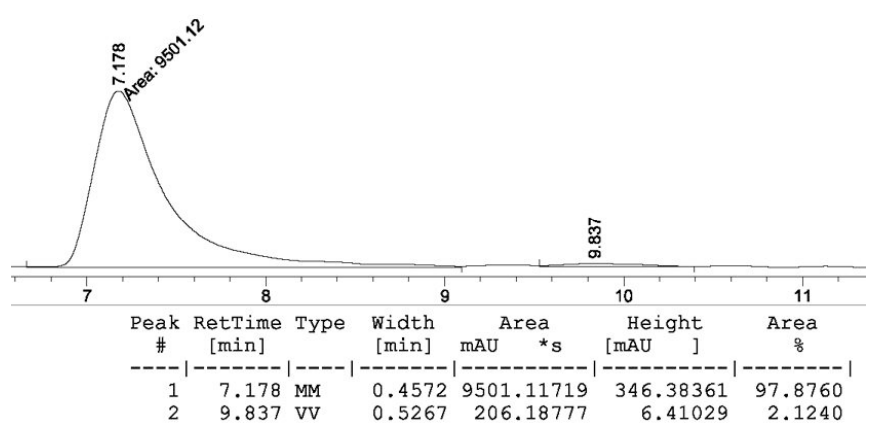


17 (Bisbenzoyl derivative employed). Chiralpak AD-H, 7.5\% i-PrOH/hexane, $1.0 \mathrm{~mL} / \mathrm{min}, 220 \mathrm{~nm}$<smiles>C=CCCN(CCC=C)C(C)(CC=C)c1ccccc1OC(=O)c1ccccc1</smiles>

Racemic

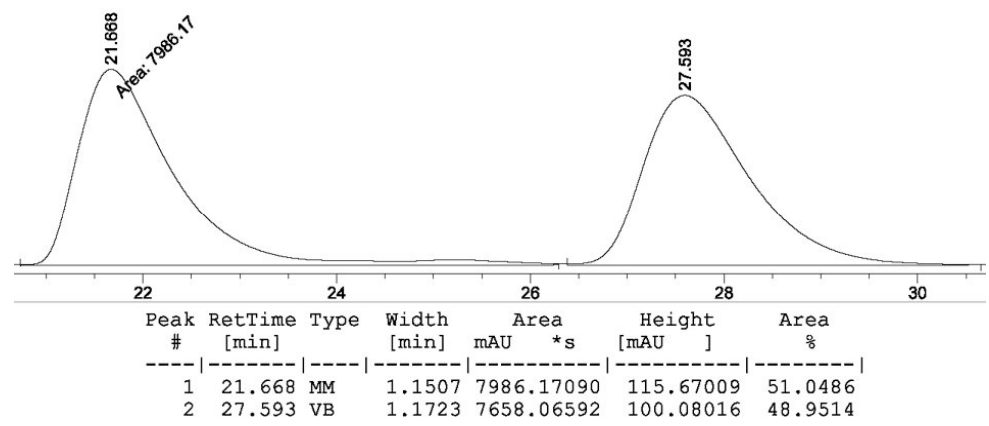

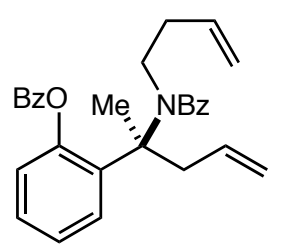

Bis-benzoyl 17 Scheme 3

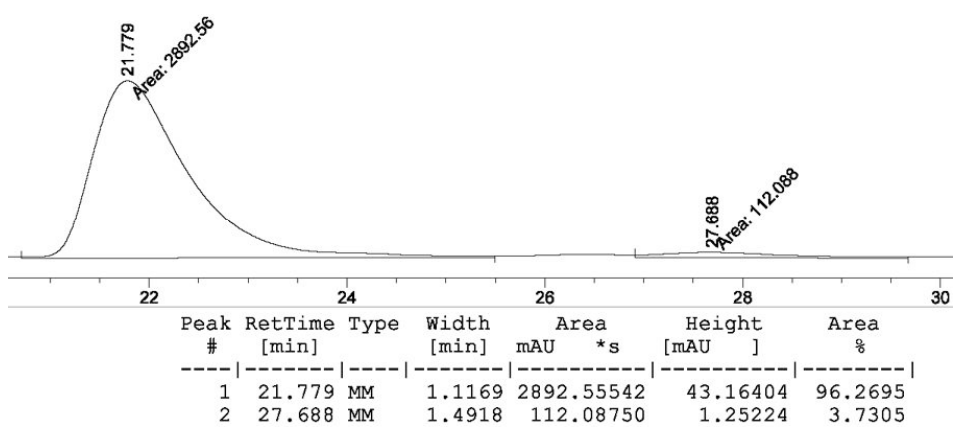

18 (Bisbenzoyl derivative employed). Chiralpak AD-H, 7.5\% i-PrOH/hexane, $1.0 \mathrm{~mL} / \mathrm{min}, 220 \mathrm{~nm}$ :<smiles>C=CCN(C(=O)c1ccccc1)[C@@](C)(c1ccccc1OC(=O)c1ccccc1)C(C)C=C</smiles>

Racemic

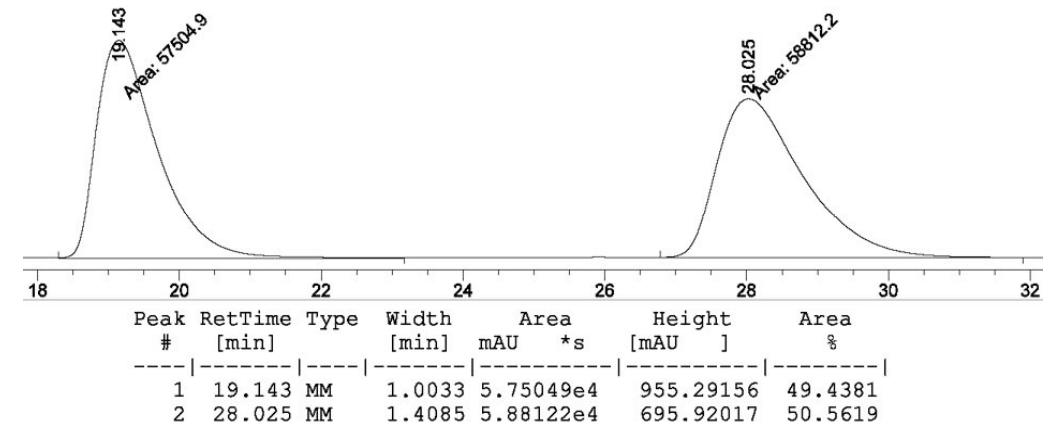<smiles>C=CCN(C(=O)c1ccccc1)[C@](C)(CC=C)c1ccccc1OC</smiles>

Bis-benzoyl 18, Scheme 3

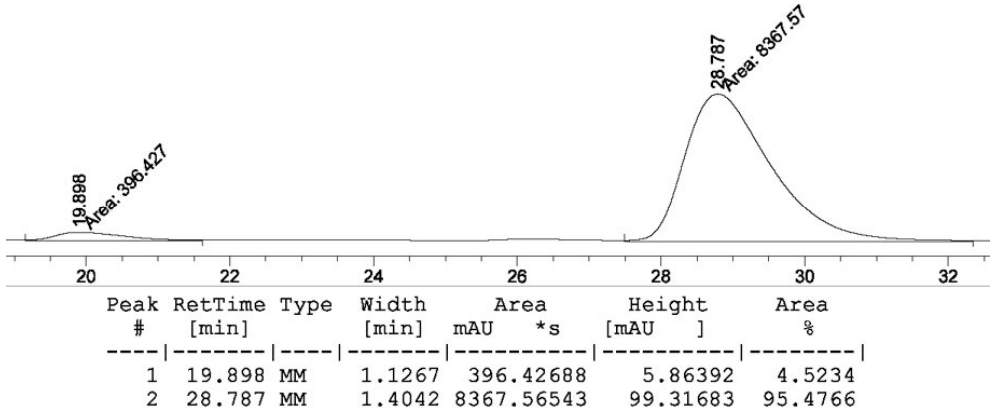


${ }^{1} \mathrm{H}$ NMR Spectra:<smiles>C=CC[C@H](Nc1c(C)cccc1O)C1CCCCC1</smiles>
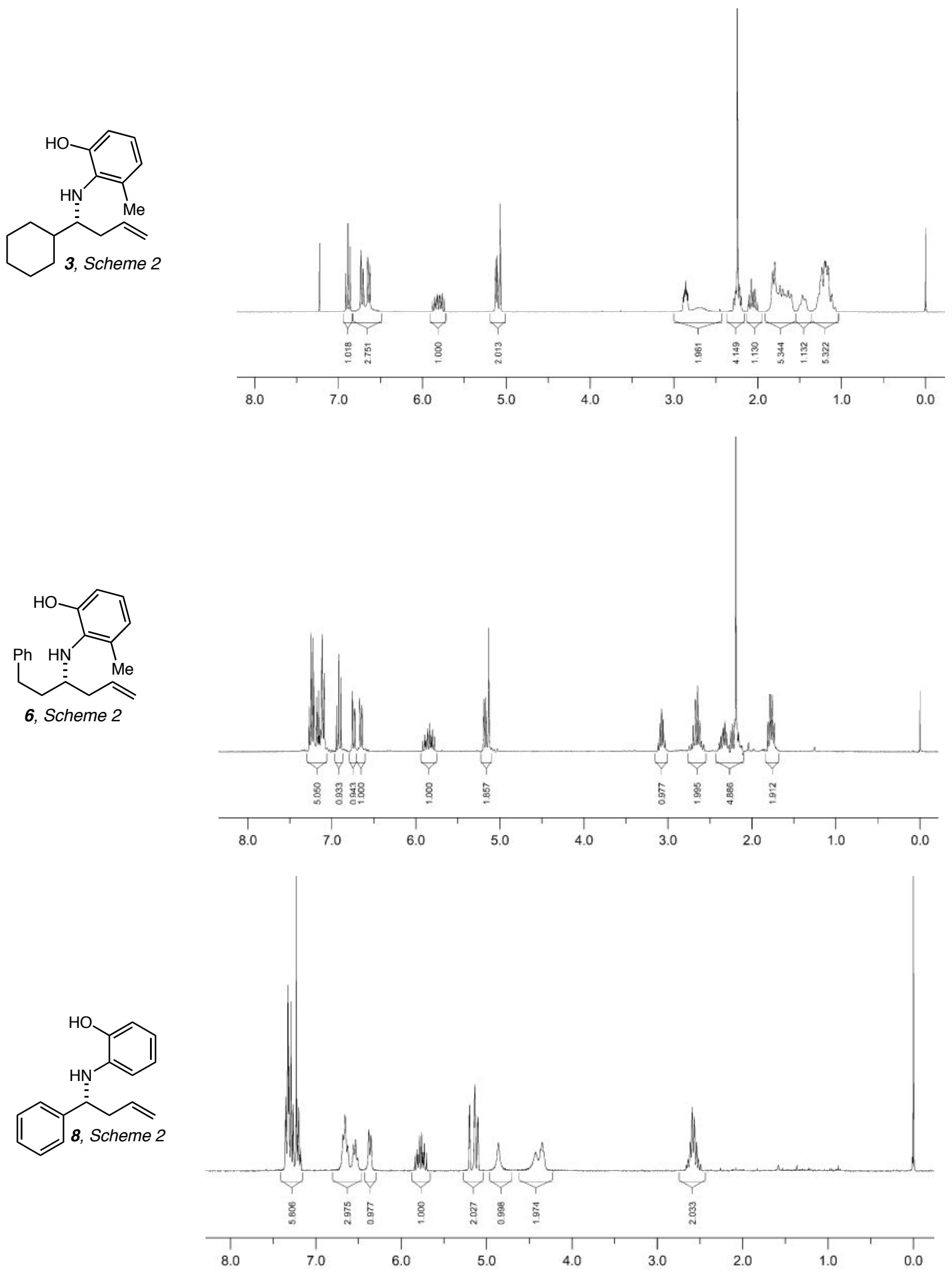
Page S15
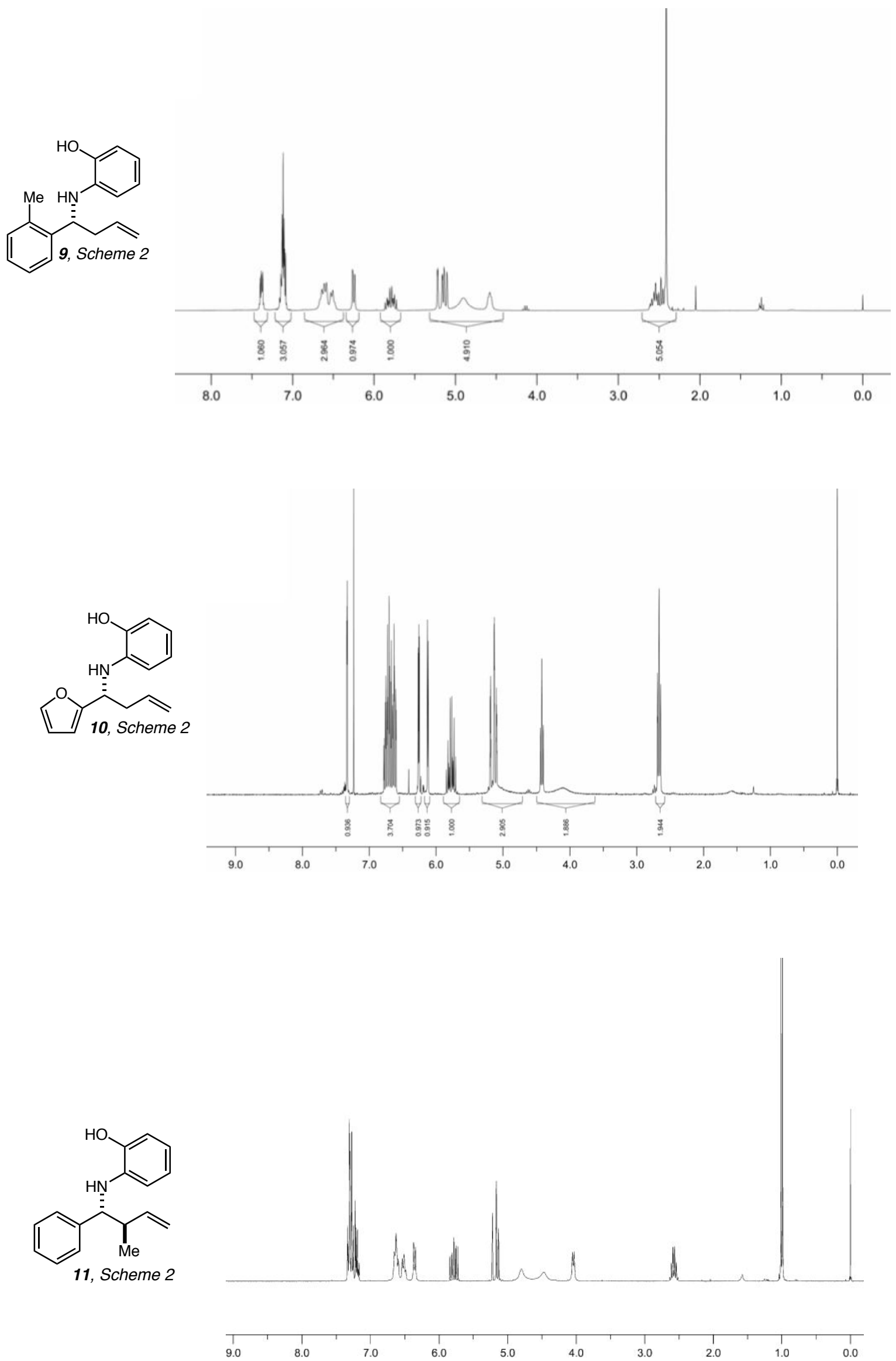
Page S16
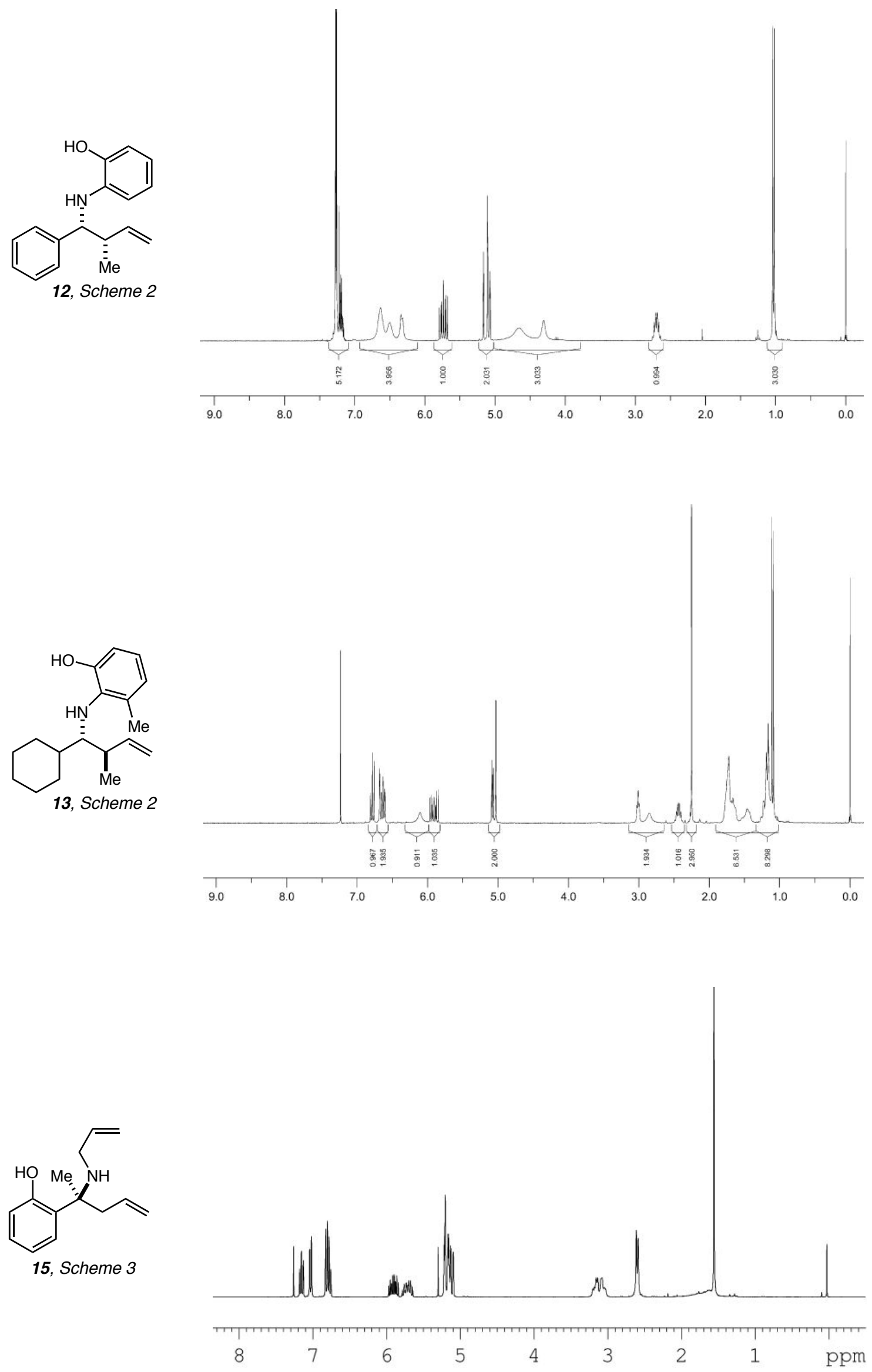
Page S17

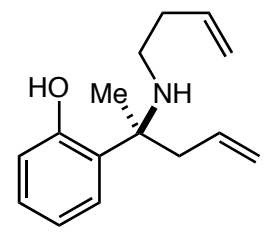

17, Scheme 3
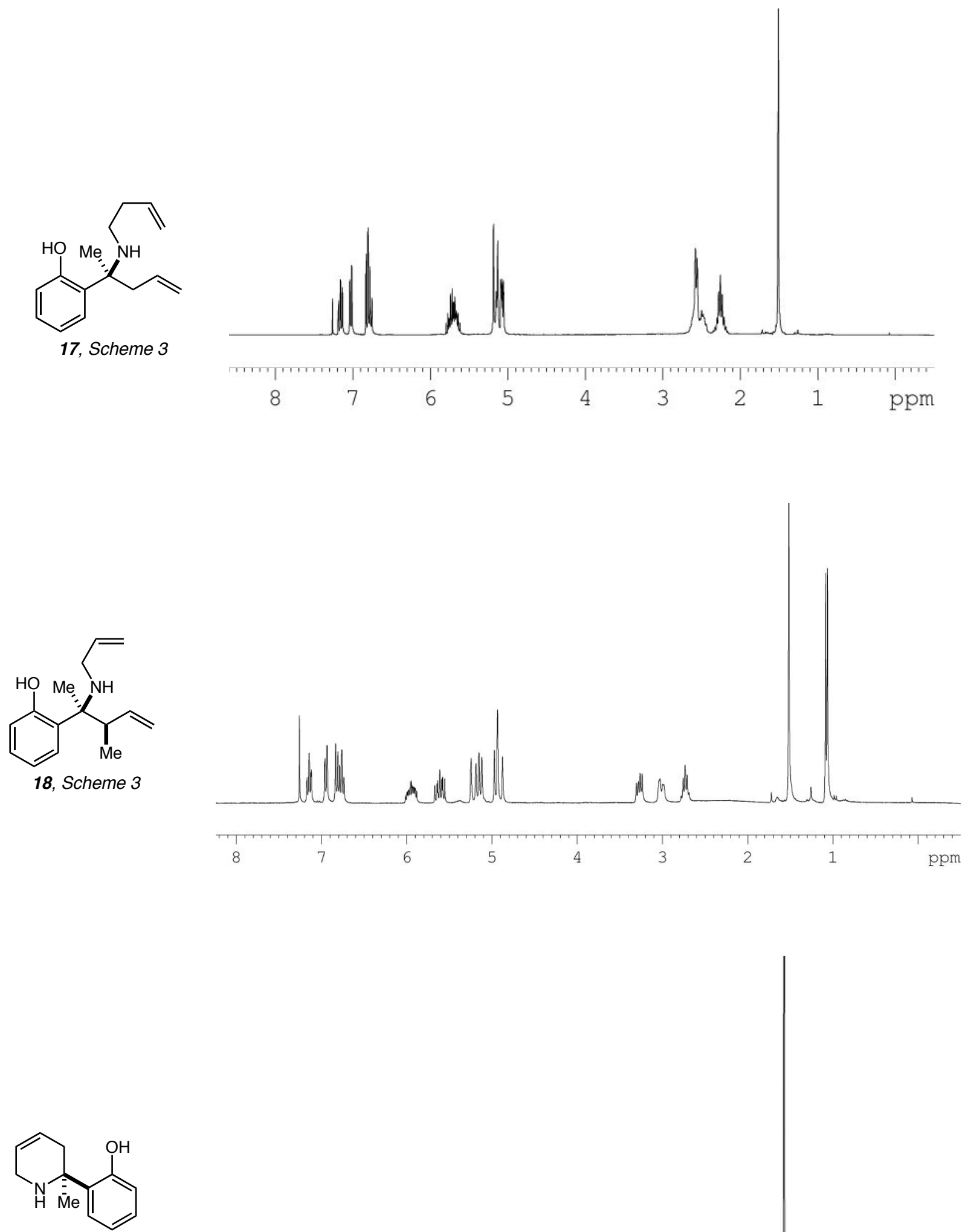

19, Scheme 4

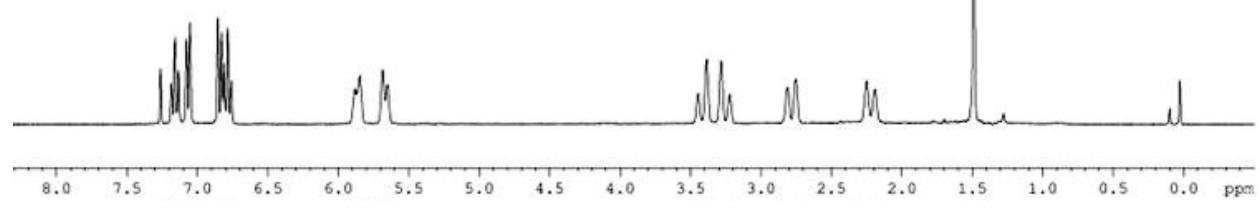




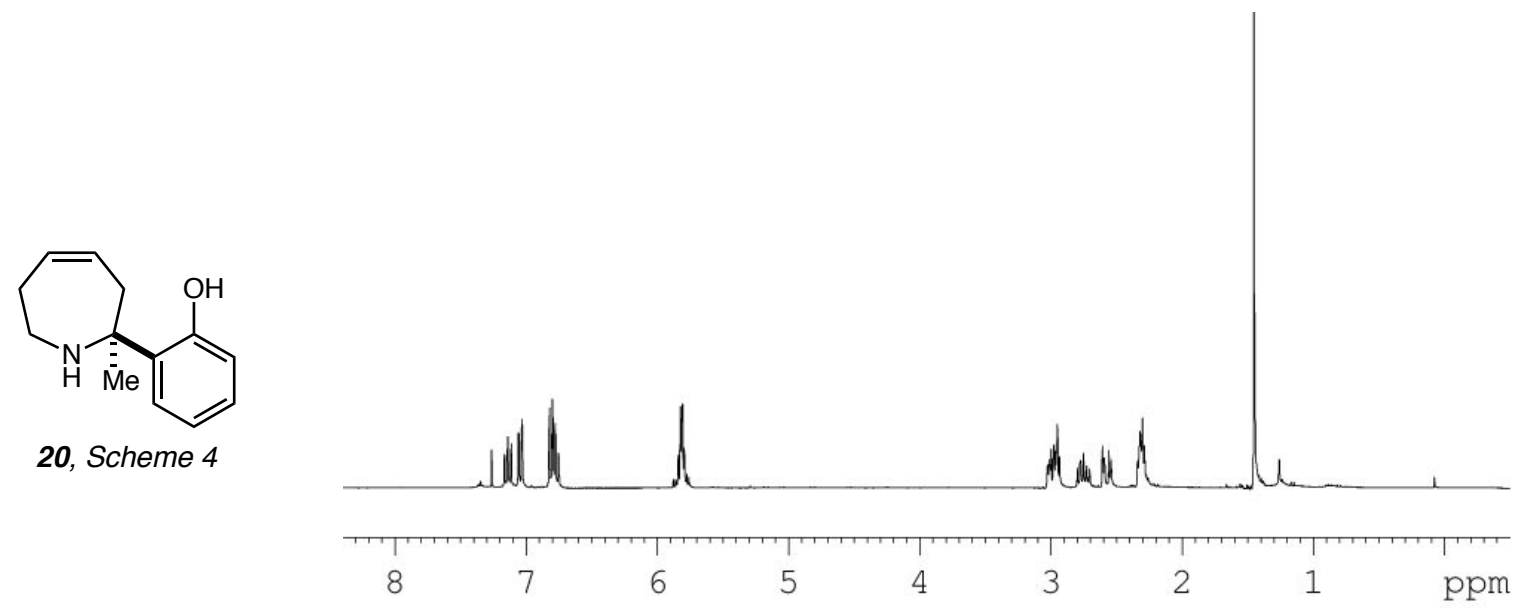

References

(1) Kobayashi, S.; Kobayashi, J.; Ishitani, H.; Ueno, M. Chem. Eur. J. 2002, 8, 4185-4190.

(2) Berger, R.; Duff, K; Leighton, J. L. J. Am. Chem. Soc. 2004, 126, 5686-5687. 